Network Working Group

Request for Comments: 2050

Obsoletes: 1466

$\mathrm{BCP}: 12$

Category: Best Current Practice
K. Hubbard

M. Kosters

InterNIC

D. Conrad

APNIC

D. Karrenberg

RIPE

J. Postel

ISI

November 1996

\title{
INTERNET REGISTRY IP ALLOCATION GUIDELINES
}

Status of this Memo

This document specifies an Internet Best Current Practices for the Internet Community, and requests discussion and suggestions for improvements. Distribution of this memo is unlimited.

IESG Note:

By approving this document as a Best Current Practice, the IESG asserts its belief that this policy described herein is an accurate representation of the current practice of the IP address registries with respect to address assignment. This does not constitute endorsement or recommendation of this policy by the IESG. The IESG will reevaluate its approval of this document in December 1997 taking into consideration the results of the discussions that will be take place in the IRE Working Group between now and then.

Abstract

This document describes the registry system for the distribution of globally unique Internet address space and registry operations. Particularly this document describes the rules and guidelines governing the distribution of this address space.

This document describes the IP assignment policies currently used by the Regional Registries to implement the guidelines developed by the IANA. The guidelines and these policies are subject to revision at the direction of the IANA. The registry working group (IRE WG) will be discussing these issues and may provide advice to the IANA about possible revisions.

This document replaces RFC 1466, with all the guidelines and procedures updated and modified in the light of experience. 
This document does not describe private Internet address space and multicast address space. It also does not describe regional and local refinements of the global rules and guidelines.

This document can be considered the base set of operational guidelines in use by all registries. Additional guidelines may be imposed by a particular registry as appropriate.

Table of Contents

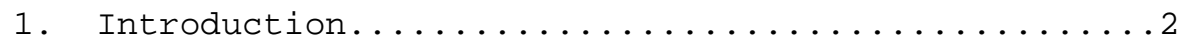

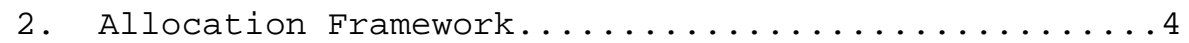

2.1 Guidelines for Internet Service Providers.......4

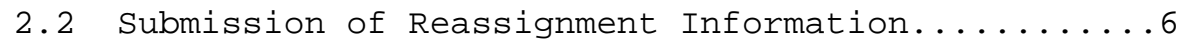

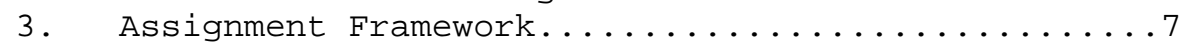

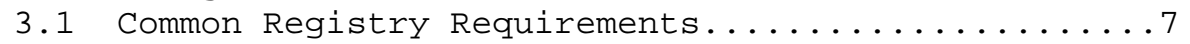

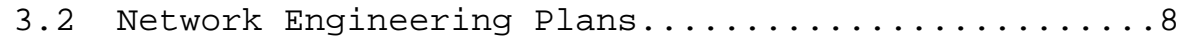

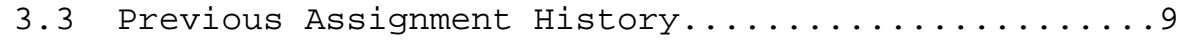

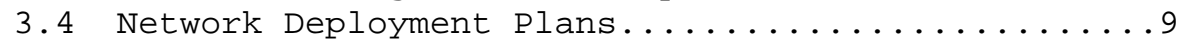

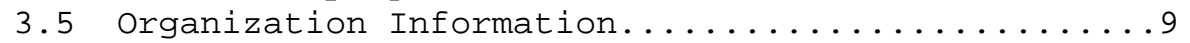

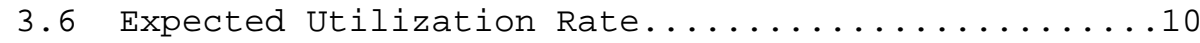

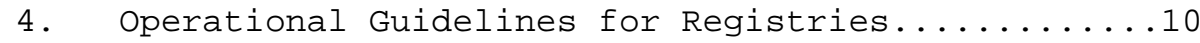

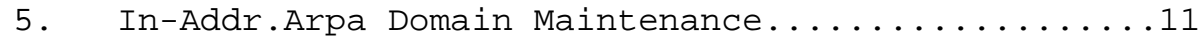

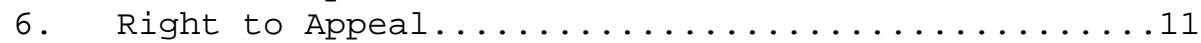

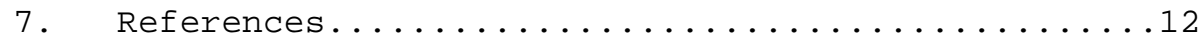

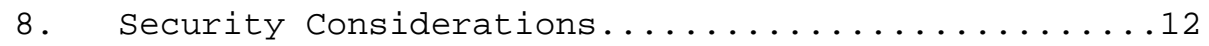

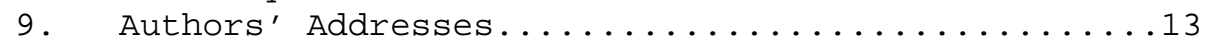

1. Introduction

The addressing constraints described in this document are largely the result of the interaction of existing router technology, address assignment, and architectural history. After extensive review and discussion, the authors of this document, the IETF working group that reviewed it and the IESG have concluded that there are no other currently deployable technologies available to overcome these limitations. In the event that routing or router technology develops to the point that adequate routing aggregation can be achieved by other means or that routers can deal with larger routing and more dynamic tables, it may be appropriate to review these constraints.

Internet address space is distributed according to the following three goals:

1) Conservation: Fair distribution of globally unique Internet address space according to the operational needs of the end-users and Internet Service Providers operating networks using this address space. Prevention of stockpiling in order to maximize the lifetime of the 
Internet address space.

2) Routability: Distribution of globally unique Internet addresses in a hierarchical manner, permitting the routing scalability of the addresses. This scalability is necessary to ensure proper operation of Internet routing, although it must be stressed that routability is in no way guaranteed with the allocation or assignment of IPv4 addresses.

3) Registration: Provision of a public registry documenting address space allocation and assignment. This is necessary to ensure uniqueness and to provide information for Internet trouble shooting at all levels.

It is in the interest of the Internet community as a whole that the above goals be pursued. However it should be noted that "Conservation" and "Routability" are often conflicting goals. All the above goals may sometimes be in conflict with the interests of individual end-users or Internet service providers. Careful analysis and judgement is necessary in each individual case to find an appropriate compromise.

The Internet Registry system

In order to achieve the above goals the Internet Registry (IR) hierarchy was established.

The Internet Registry hierarchy consists of the following levels of hierarchy as seen from the top down: IANA, Regional IRs, Local IRS.

IANA

The Internet Assigned Numbers Authority has authority over all number spaces used in the Internet. This includes Internet Address Space. IANA allocates parts of the Internet address space to regional IRs according to its established needs.

Regional IRs

Regional IRs operate in large geopolitical regions such as continents. Currently there are three regional IRs established; InterNIC serving North America, RIPE NCC serving Europe, and APNIC serving the Asian Pacific region. Since this does not cover all areas, regional IRs also serve areas around its core service areas. It is expected that the number of regional IRs will remain relatively small. Service areas will be of continental dimensions. 
Regional IRs are established under the authority of the IANA. This requires consensus within the Internet community of the region. A consensus of Internet Service Providers in that region may be necessary to fulfill that role.

The specific duties of the regional IRs include coordination and representation of all local IRs in its respective regions.

Local IRs

Local IRs are established under the authority of the regional IR and IANA. These local registries have the same role and responsibility as the regional registries within its designated geographical areas. These areas are usually of national dimensions.

\section{Allocation Framework}

\subsection{Guidelines for Internet Service Providers (ISPS)}

This document makes a distinction between the allocation of IP addresses and the assignment of IP addresses. Addresses are allocated to ISPs by regional registries to assign to its customer base.

ISPs who exchange routing information with other ISPs at multiple locations and operate without default routing may request space directly from the regional registry in its geographical area. ISPS with no designated regional registry may contact any regional registry and the regional registry may either handle the request or refer the request to an appropriate registry.

To facilitate hierarchical addressing, implemented using Classless Inter-Domain Routing (CIDR), all other ISPs should request address space directly from its upstream provider. ISPs only request address space directly from regional registries if their immediate requirement, when satisfied with a contiguous block allocation, has a reasonable probability of being routable on the Internet, and they meet one or more of the following conditions.

a) the ISP is directly connected to a major routing exchange (for purposes of this document, a major routing exchange is defined as a neutral layer 2 exchange point connecting four or more unrelated ISPs.)

b) the ISP is multi-homed, that is, it has more than one simultaneous connection to the global Internet and no connection is favored over the other 
Note that addresses issued directly from the IRs (non-provider based), are the least likely to be routable across the Internet.

The following are the IP allocation guidelines for ISPs:

1. CIDR addresses are allocated to ISPs in blocks. It is recommended that those blocks remain intact. Fragmentation of CIDR blocks is discouraged. More specifically, ISPs are encouraged to treat address assignments as loans for the duration of the connectivity provision. At the termination of the Internet connectivity contract, e.g., the customer moves to another service provider, it is recommended the customer return the network addresses currently in use and renumber into the new provider's address space. The ISP should allow sufficient time for the renumbering process to be completed before the IP addresses are reused.

2. To ensure efficient implementation and use of Classless Inter-Domain Routing (IDR), the Regional Registries issue address space on appropriate "CIDR-supported" bit boundaries.

3. ISPs are required to utilize address space in an efficient manner. To this end, ISPs should have documented justification available for each assignment. The regional registry may, at any time, ask for this information. If the information is not available, future allocations may be impacted. In extreme cases, existing loans may be impacted.

4. IP addresses are allocated to ISPs using a slow-start procedure. New ISPS will receive a minimal amount based on immediate requirement. Thereafter, allocated blocks may be increased based on utilization verification supplied to the regional registry. The parent registries are responsible for determining appropriate initial and subsequent allocations. Additional address allocations will provide enough address space to enable the ISP to assign addresses for three months without requesting additional address space from its parent registry. Please note that projected customer base has little impact on the address allocations made by the parent registries. Initial allocation will not be based on any current or future routing restrictions but on demonstrated requirements.

5. Due to the requirement to increase the utilization efficiency of IPv4 address space, all assignments are made with the assumption that sites make use of variable length subnet mask (VLSM) and classless technologies within their network. Any request for address space based on the use of classfull 
assumptions will require a detailed justification. The use of classfull technologies for the purposes of administrative convenience is generally insupportable due to the limited availability of free IPv4 address space.

6. Regional registries may set a maximum limit on assignment sizes such that a second opinion of the regional registry is required.

7. Due to constraints on the available free pool of IPv4 address space, the use of static IP address assignments (e.g., one address per customer) for dial-up users is strongly discouraged. While it is understood that the use of static addressing may ease some aspects of administration, the current rate of consumption of the remaining unassigned IPV4 address space does not permit the assignment of addresses for administrative ease. Organizations considering the use of static IP address assignment are expected to investigate and implement dynamic assignment technologies whenever possible.

\subsection{Submission of Reassignment Information}

It is imperative that reassignment information be submitted in a prompt and efficient manner to facilitate database maintenance and ensure database integrity. Therefore, assignment information must be submitted to the regional registry immediately upon making the assignment. The following reasons necessitate transmission of the reassignment information:

a) to provide operational staff with information on who is using the network number and to provide a contact in case of operational/security problems,

b) to ensure that a provider has exhausted a majority of its current CIDR allocation, thereby justifying an additional allocation,

c) to assist in IP allocation studies.

Procedures for submitting the reassignment information will be determined by each regional registry based on its unique requirements.

All sub-registries (ISPs, Local registries, etc.) must register with their respective regional registry to receive information regarding reassignment guidelines. No additional CIDR blocks will be allocated by the regional registry or upstream providers until approximately 80\% of all reassignment information has been submitted. 


\section{Assignment Framework}

An assignment is the delegation of authority over a block of IP addresses to an end enterprise. The end enterprise will use addresses from an assignment internally only; it will not subdelegate those addresses. This section discusses some of the issues involved in assignments and the framework behind the assignment of addresses.

In order for the Internet to scale using existing technologies, use of regional registry services should be limited to the assignment of IP addresses for organizations meeting one or more of the following conditions:

a) the organization has no intention of connecting to the Internet-either now or in the future-but it still requires a globally unique IP address. The organization should consider using reserved addresses from RFC1918. If it is determined this is not possible, they can be issued unique (if not Internet routable) IP addresses.

b) the organization is multi-homed with no favored connection.

c) the organization's actual requirement for IP space is very large, for example, the network prefix required to cover the request is of length /18 or shorter.

All other requestors should contact its ISP for address space or utilize the addresses reserved for non-connected networks described in RFC1918 until an Internet connection is established. Note that addresses issued directly from the IRs, (non-provider based), are the least likely to be routable across the Internet.

\subsection{Common Registry Requirements}

Because the number of available IP addresses on the Internet is limited, the utilization rate of address space will be a key factor in network number assignment. Therefore, in the best interest of the Internet as a whole, specific guidelines have been created to govern the assignment of addresses based on utilization rates.

Although topological issues may make exceptions necessary, the basic criteria that should be met to receive network numbers are listed below:

25\% immediate utilization rate

$50 \%$ utilization rate within 1 year 
The utilization rate above is to be used as a guideline, there may be be occasions when the 1 year rate does not fall exactly in this range. Organizations must exhibit a high confidence level in its 1 year utilization rate and supply documentation to justify the level of confidence.

Organizations will be assigned address space based on immediate utilization plus 1 year projected utilization. A prefix longer than 124 may be issued if deemed appropriate. Organizations with less than 128 hosts will not be issued an IP address directly from the IRs. Organizations may be issued a prefix longer than /24 if the organization can provide documentation from a registry recognized ISP indicating the ISP will accept the long prefix for injection into the global routing system.

Exceptions to the criteria will not be made based on insufficient equipment without additional detailed justification. Organizations should implement variable length subnet mask (VLSM) internally to maximize the effective utilization of address space. Address assignments will be made under the assumption that VLSM is or will be implemented.

IP addresses are valid as long as the criteria continues to be met. The IANA reserves the right to invalidate any IP assignments once it is determined the the requirement for the address space no longer exists. In the event of address invalidation, reasonable efforts will be made by the appropriate registry to inform the organization that the addresses have been returned to the free pool of IPv4 address space.

\subsection{Network Engineering Plans}

Before a registry makes an assignment, it must examine each address space request in terms of the requesting organization's networking plans. These plans should be documented, and the following information should be included:

1. subnetting plans, including subnet masks and number of hosts on each subnet for at least one year

2. a description of the network topology

3. a description of the network routing plans, including the routing protocols to be used as well as any limitations. 
The subnetting plans should include:

a) a tabular listing of all subnets on the network

b) its associated subnet masks

c) the estimated number of hosts

d) a brief descriptive remark regarding the subnet.

If subnetting is not being used, an explanation why it cannot be implemented is required. Care must be taken to ensure that the host and subnet estimates correspond to realistic requirements and are not based on administrative convenience.

\subsection{Previous Assignment History}

To promote increased usage of address space, the registries will require an accounting of address space previously assigned to the enterprise, if any. In the context of address space allocation, an "enterprise" consists of all divisions and/or subsidiaries falling under a common parent organization. The previous assignment history should include all network numbers assigned to the organization, plus the network masks for those networks and the number of hosts on each (sub-) network. Sufficient corroborating evidence should be provided to allow the assigning registry to be confident that the network descriptions provided are accurate. Routing table efficiency will be taken into account by the regional registries and each request will be handled on a case by case basis.

\subsection{Network Deployment Plans}

In order to assign an appropriate amount of space in the required time frame, a registry may request deployment plans for a network. Deployment plans should include the number of hosts to be deployed per time period, expected network growth during that time period, and changes in the network topology that describe the growth.

\subsection{Organization Information}

A registry may request that an organization furnish a published description verifying that the organization is what it claims to be. This information can consist of brochures, documents of incorporation, or similar published material. 


\subsection{Expected Utilization Rate}

As stated in the foregoing text, one of the key factors in determining how much address space is appropriate for an organization is the expected utilization rate of the network. The expected utilization rate is the number of hosts connected to the network divided by the total number of hosts possible on the network. In addition, the estimated number of hosts should be projected over a reasonable time frame, i.e., one in which the requesting enterprise has a high level of confidence. The minimal utilization rate is set by the IANA and may be changed at any time. New utilization rates may be enforced by the regional registries prior to updating the written policy.

4. Operational Guidelines For Registries

1. Regional Registries provide registration services as its primary function. Therefore, regional registries may charge some fee for services rendered, generally in relation to the cost of providing those services.

2. Regardless of the source of its address space, sub-registries (Local IRs, ISPs, etc.) must adhere to the guidelines of its regional registry. In turn, it must also ensure that its customers follow those guidelines.

3. To maximize the effective use of address space, IP addresses need to be assigned/allocated in classless blocks. With this in mind, assignments will not be made in Class Cs or Bs but by prefix length. Consequently, an organization that would have been assigned a Class $B$ in the past will now be assigned a /16 prefix, regardless of the actual address class.

4. All IP address requests are subject to audit and verification by any means deemed appropriate by the regional registry. If any assignment is found to be based on false information, the registry may invalidate the request and return the assigned addresses back to the pool of free addresses for later assignment.

5. Due to technical and implementation constraints on the Internet routing system and the possibility of routing overload, major transit providers may need to impose certain restrictions to reduce the number of globally advertised routes. This may include setting limits on the size of CIDR prefixes added to the routing tables, filtering of non-aggregated routes, etc. Therefore, addresses obtained directly from regional registry (provider-independent, also known as portable) are not 
guaranteed routable on the Internet.

6. Information provided to request address space is often considered sensitive by the requesting organization. The assigning registry must treat as confidential any and all information that the requesting organization specifically indicates as sensitive. When a requesting organization does not have assurance of privacy, the parent of the assigning registry may be required to do the assignment. In such cases, the parent registry will provide the assigning registry with information regarding the appropriate amount of address space to allocate.

7. The transfer of IP addresses from one party to another must be approved by the regional registries. The party trying to obtain the IP address must meet the same criteria as if they were requesting an IP address directly from the IR.

5. In-ADDR.ARPA Domain Maintenance

The regional registries will be responsible for maintaining INADDR.ARPA records only on the parent blocks of IP addresses issued directly to the ISPs or those CIDR blocks of less than /16. Local IRs/ISPS with a prefix length of /16 or shorter will be responsible for maintaining all IN-ADDR.ARPA resource records for its customers.

IN-ADDR.ARPA resource records for networks not associated with a specific provider will continue to be maintained by the regional registry.

6. Right to Appeal

If an organization feels that the registry that assigned its address has not performed its task in the requisite manner, the organization has the right of appeal to the parent registry.

In such cases, the assigning registry shall make available all relevant documentation to the parent registry, and the decision of the parent registry shall be considered final (barring additional appeals to the parent registry's parent). If necessary, after exhausting all other avenues, the appeal may be forwarded to IANA for a final decision. Each registry must, as part of their policy, document and specify how to appeal a registry assignment decision. 
7. References

[RFC 1519] Fuller, V., Li, T., Yu, J., and K. Varadhan, "Classless Inter- Domain Routing (CIDR) : an Address Assignment and Aggregation Strategy", September 1993.

[RFC 1518] Rekhter, Y., and T. Li, "An Architecture for IP Address Allocation with CIDR", September 1993.

[RFC 1918] Rekhter, Y., Moskowitz, B., Karrenberg, D., and G. de Groot, "Address Allocation for Private Internets", February 1996 .

[RFC 1814] Gerich, E., "Unique Addresses are Good", June 1995.

[RFC 1900] Carpenter, B., and Y. Rekhter, "Renumbering Needs Work", February 1996.

8. Security Considerations

Security issues are not discussed in this memo. 
9. Authors' Addresses

Kim Hubbard

InterNIC Registration Services

c/o Network Solutions

505 Huntmar Park Drive

Herndon, VA 22070

Phone: (703) 742-4870

EMail: kimh@internic.net

Mark Kosters

InterNIC Registration Services

c/o Network Solutions

505 Huntmar Park Drive

Herndon, VA 22070

Phone: (703) 742-4795

EMail: markkeinternic.net

David Conrad

Asia Pacific Network Information Center

c/o United Nations University

53-70 Jingumae 5-chome,

Shibuya-ku, Tokyo 150

JP

Phone: +81-3-5467-7014

EMail: davidc@APNIC.NET

Daniel Karrenberg

RIPE NCC

Kruislaan 409

SJ Amsterdam NL-1098

$\mathrm{NL}$

Phone: +31 205925065

EMail: dfk@RIPE.NET

Jon Postel

USC/Information Sciences Institute

4676 Admiralty Way

Marina del Rey, CA 90292

Phone: 310-822-1511

EMail: PosteleISI.EDU

Hubbard, et. al.

Best Current Practice

[Page 13] 